\title{
Body Attitudes and Eating Behaviours of Female Malay Students Studying in Australia
}

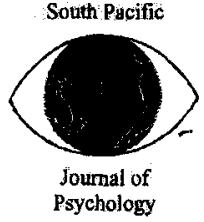

Andrew J. McDowell and Malcolm J. Bond

Flinders University

Adelaide, Australia

\begin{abstract}
The extent to which 113 adolescent Malay women studying in Australia expressed the desire to lose weight was examined, as was the degree to which this desire reflected a genuine need to lose weight. Comparisons between participants who expressed the desire to either lose weight, stay at their current weight, or gain weight were made using both attitudinal and behavioural components of body image. An in-depth analysis of those who wished to lose weight was also undertaken. There was evidence that negative body attitudes were in excess of what actual body weight might dictate. However, these negative attitudes were not found to be consistently predictive of dysfunctional eating behaviours. Results were interpreted with reference to the potential influence of Westernisation in countries in the South Pacific region, and in particular how attitudes and behaviours are influenced by this process.
\end{abstract}

\section{Body Attitudes and Eating Behaviours of Female Malay Students Studying in Australia}

Dissatisfaction with weight and shape (negative body image) has become so common that it has been termed a normative discontent (Rodin, Silberstein \& Striegel-Moore, 1985). This dissatisfaction does not only manifest among those who are objectively overweight; rather it can be identified in individuals across all weight ranges (Crawford \& Worsley, 1988). Although the prevalence of negative body image is much higher among women, it is also identifiable among men (Rosen \& Gross, 1987). Discontent with weight and shape also has no age barriers, with data from participants younger than 10 years (Rumpel \& Harris, 1994) and older than 90 years (Rand \& Kuldau, 1990; Stevens, Kumanyika \& Keil, 1994) being illustrative of the phenomenon.

What is considered a desirable weight or shape varies between cultures and over time. Indeed, it is now accepted that body image disturbance is socio-culturally determined (Banks, 1992; Dolan, 1991; Furnham \& Baguma, 1994; Heinberg, 1996; Pate, Pumariega, Hester \& Garner, 1992; Powell \& Kahn, 1995), with a particularly high prevalence in Western countries (Altabe, 1996; Indran \& Hatta, 1995; Lee, 1994, 1996). This has led Vandereycken (1993) to describe Western cultures as 'lipophobic'. An emerging issue is the degree to which this body-conscious Western ideal has pervaded non-Western cultures.

Like many South-East Asian countries, Malaysia has experienced significant economic and social change over the past twenty years that has brought with it an increasing level of Westernisation (Rahim, 1998; Robinson \& Goodman, 1996). The influence of Western media, particularly television, has resulted in a decline in national identity and the broad absorption of Western ideals and images (Kling, 1995; Nain, 1996). This has produced a conflict between traditional cultural expectations and aspirations based on Western notions of 
success. In Malaysia, this adaptive challenge has resulted in psychosocial distress such as anomie (Ariffin, 1995) and some backlash against the automatic acceptance of Western values (Kahn, 1997). Further, Littlewood (1995) warns that the ambiguous shift to 'modernity' that is apparent in Malaysia is likely to be associated with the loss of adaptive instrumental strategies when faced with the ubiquity of potentially unlimited consumption.

The food ideology and eating behaviour of Malaysians are complex, being influenced by both culture and religion (Laderman, 1984). Indran and Hatta (1995) have suggested that these traditional socio-cultural influences guide the eating attitudes and behaviours of Malaysians and may actually negate the otherwise pervasive Western pursuit of thinness. They conclude that the pressure to conform to Western ideals of body image is only modest. In fact, there is evidence that the availability of Western-style food is having a contrary effect. While Malaysian samples traditionally have a lower average Body Mass Index (BMI; Garrow \& Webster, 1985) than do, for example, Australian samples, recent studies have shown a tendency toward obesity (Hughes et al., 1990). For example, Lim, Liam, $\mathrm{Ng}$ and Teow (1994) examined National Service archives from the Singapore/Malaysia region and found that the proportion of male recruits with a BMI in the overweight range $\left(>25 \mathrm{~kg} / \mathrm{m}^{2}\right)$ increased from $1.37 \%$ in 1967 to $12.07 \%$ in 1991 .

In relation to the inappropriate pursuit of thinness, only a few studies of body image disturbance in Malaysia have been reported. Buhrich (1981) surveyed the frequency of presentation of anorexia nervosa among psychiatrists working in Peninsula Malaysia. From approximately 60,000 new cases surveyed, only 30 were diagnosed with anorexia nervosa ( 28 females and two males). While this is a very low proportion, it was noted that these patients tended to be from higher social classes that had been more exposed to Western ideals. A later study (Goh, Ong \& Subramaniam, 1993) examined the admissions to psychiatric wards at the University of Malaya between 1970 and 1988 . No eating disorder cases were identified from approximately 8,000 male admissions, while there were only 15 cases of anorexia nervosa from among 9,000 female admissions. More recently, Schmidt (1993) documented the case of a Malaysian male with bulimia nervosa and proposed that conflict about socio-cultural norms played an important part in the onset and maintenance of his eating disorder. It is difficult to draw any conclusions about body image disturbance in Malaysia from these modest studies. Obvious difficulties include the fact that the data are relatively old, that the studies were limited by their retrospective methodology, and that members of different Malay ethnic groups are often aggregated within the one sample. Further, it is likely that many patients with symptoms of disordered eating would have consulted traditional healers rather than psychiatrists (Buhrich, 1981).

More recent and methodologically rigorous research conducted in the Asian region suggests that eating attitudes and behaviours related to fear of fatness are indeed problematic. For example, both psychological and behavioural concomitants of body image disturbance are now commonly found in young females from high-income societies such as Japan, Singapore, Hong Kong, Taiwan and the Republic of Korea (Kiriike, Nagata, Sirata \& Yamamoto, 1998; Lee, 1998; Lee, Leung, Lee, Yu \& Leung, 1996; Lippincott \& Hwang, 1999; Nogami, 1997). Of most concern is that while such young females are generally of normal weight, they still desire to be thinner. For example, while the median BMI of Chinese female undergraduate students in Hong Kong is $19.3 \mathrm{~kg} / \mathrm{m}^{2}, 70 \%$ of them report a desire to be "a little slimmer" (Lee et al., 1996).

The effects of negative body image may range from sub-clinical symptoms such as a general negative body image to severe clinical illnesses such as anorexia and bulimia (Button \& 
Whitehouse, 1981). Prevention of the progression to clinical illness is ultimately more desirable than cure (Felts, Parrillo, Chenier \& Dunn, 1996), particularly as Lee (1998) raises concerns about the ability of Asian health systems to practically address clinical problems of eating disorders. It is therefore vital to understand the potentially predisposing attitudes and behaviours that are prevalent in the broader community through the study of non-clinical samples (Kenny \& Adams, 1994; Maude, Wertheim, Paxton, Gibbons \& Szmukler, 1993; Patton, Johnson-Sabine, Wood, Mann \& Wakeling, 1990).

In acknowledgment of these comments, the current study explored the extent to which there was an expressed desire to lose weight among a sample of Malaysian women, from the Malay ethnic group, who are studying in Australia. The degree to which such a desire reflected a genuine need to lose weight, as opposed to being driven by psychological concerns about body image, was also examined. Both attitudinal and behavioural components of body image were measured. The goal was to determine whether the current sample exhibited cognitive and affective states that might predispose to negative body image and/or manifested dissatisfaction with weight and shape in dysfunctional eating behaviours.

\section{Method}

\section{Participants and Procedure}

The sample comprised 113 female students from the Malay ethnic group who were recent arrivals to Australia. Their purpose was to attend a bridging course designed to develop their study skills prior to taking up positions at Australian universities. Data were collected as part of a teaching session on social research methods. Although participation was voluntary, a $100 \%$ response rate was obtained. The mean age was 20.4 years $(\mathrm{SD}=0.6$ years). Approval was gained from the Social and Behavioural Research Ethics Committee of Flinders University, and participants were treated in accordance with the principles of ethical research of the Australian Psychological Society (1994). Data management and analysis were conducted using SPSS for Windows (Norušis, 1993). An initial aim of the study was to seek information concerning issues such as religion and family income. However, permission for these inquiries was denied as they were considered to be unnecessary to the core objectives of the study and potentially culturally insensitive. The conduct of the study was not contiguous with Ramadan.

\section{Questionnaire}

\section{Weight And Weight Control}

Height, current weight and desired weight were used to derive actual and desired BMI. Perception of current weight was sought using a 5-point scale from 'very underweight' through 'just right' to 'very overweight'. Further questions concerned general eating habit ('undereat', 'eat sensibly', 'overeat'), whether participants consciously attempted to control their weight ('yes' / 'no'), and whether they had been on a specific diet for the control of their weight in the past 12 months ('yes'/ 'no').

\section{Body Attitudes Questionnaire (BAQ).}

The BAQ contains 44 self-referent statements to which participants respond using 5-point scales ranging from 'strongly disagree' to 'strongly agree' (Ben-Tovim \& Walker, 1991). Six body attitudes are assessed: Feelings of Overall Fatness (possible range 13-65), Selfdisparagement (8-40), Strength and Fitness (6-30), Salience of Weight and Shape (8-40), Feelings of Attractiveness (5-25), and Consciousness of Lower Body Fat (4-20). In all cases higher scores indicate greater importance of the body attitude being measured. The BAQ has 
sound psychometric properties and has some cross-cultural validity, having previously been used successfully with a Samoan sample (Wilkinson, Ben-Tovim \& Walker, 1994). Internal reliabilities of the subscales ( $\alpha$; Cronbach, 1951) with the current sample ranged from .66 for Strength and Fitness to .91 for Feelings of Overall Fatness, although Feelings of Attractiveness provided a coefficient of only .45.

\section{Three-Factor Eating Questionnaire (TFEQ).}

The TFEQ (Stunkard \& Messick, 1985) is a 51-item scale developed from the theory of restrained eating. Its sub-scales assess eating behaviour in terms of Restraint (possible range $0-21 ; \alpha=.85)$, Dis-inhibition (0-16; $\alpha=.73)$, and Hunger $(0-14 ; \alpha=.72)$. Higher scores represent the more frequent practice of restrained eating, of dis-inhibited eating, and of eating due to feeling hungry, respectively. The TFEQ has been used extensively in studies of cognitive-behavioural eating patterns (Stunkard, 1997).

\section{Results}

\section{Actual and Desired Weight}

The average BMI of the sample was $21.2(S D=2.9)$, which is towards the lower limit of the normal range $\left(20-25 \mathrm{~kg} / \mathrm{m}^{2}\right)$. In fact, 41 participants $(36 \%)$ were classified as underweight $(<$ $\left.20 \mathrm{~kg} / \mathrm{m}^{2}\right), 62(55 \%)$ were normal and only $10(9 \%)$ were overweight or obese $\left(>25 \mathrm{~kg} / \mathrm{m}^{2}\right)$. Despite this, 74 participants $(66 \%)$ indicated that they would like to lose weight (mean desired weight loss $=5.7 \mathrm{~kg}, S D=4.1$ ), with only $15(13 \%)$ desiring no weight change and 24 (21\%) suggesting they would like to gain weight (mean desired weight gain $=3.0 \mathrm{~kg}, S D=$ 1.6). The mean BMI of these subgroups is shown in Table 1. Those desiring weight loss had a significantly higher BMI than either of the other subgroups.

\section{Body Attitudes}

The body attitude measures derived from the BAQ are presented in Table 1, according to whether weight loss, weight gain, or no weight change was desired. Participants who desired weight loss had significantly more negative attitudes toward their bodies than those who desired weight gain in terms of Feelings of Overall Fatness, Self-disparagement, Salience of Weight and Shape, and Consciousness of Lower Body Fat. They were also significantly more negative than participants desiring no weight change in Feelings of Overall Fatness and Salience of Weight and Shape.

\section{Eating Behaviours}

Participants desiring a weight loss reported significantly higher levels of Restraint than those desiring a weight gain. No significant pair-wise differences were noted for disinhibition, while participants desiring a weight gain reported significantly higher levels of Hunger than those desiring no change in weight. These results are also presented in Table 1. 
Table 1

Comparisons of Participants Desiring Weight Loss, No Change or Weight Gain

\begin{tabular}{|c|c|c|c|c|c|c|c|}
\hline & \multicolumn{2}{|c|}{$\begin{array}{l}\text { Weight loss } \\
\text { desired }\end{array}$} & \multicolumn{2}{|c|}{$\begin{array}{l}\text { No change } \\
\text { desired }\end{array}$} & \multicolumn{2}{|c|}{$\begin{array}{l}\text { Weight gain } \\
\text { desired }\end{array}$} & \multirow[b]{2}{*}{$F$} \\
\hline & $M$ & $(S D)$ & $M$ & $(S D)$ & $M$ & $(S D)$ & \\
\hline Current BMI & $22.6_{a}$ & (2.4) & $19.4_{b}$ & $(1.2)$ & $18.0_{b}$ & $(1.3)$ & $48.62^{* * *}$ \\
\hline \multicolumn{8}{|l|}{ Body attitudes } \\
\hline Feelings of Overall Fatness & 46.4 & $(6.7)$ & $33.9 \mathrm{~b}$ & (6.1) & $29.0_{\mathrm{b}}$ & $(6.0)$ & $75.14^{* * * *}$ \\
\hline Self-disparagement & $17.2 \mathrm{a}$ & (3.6) & 14.9 & (3.9) & $14.6 \mathrm{~b}$ & $(2.9)$ & $6.28^{* *}$ \\
\hline Strength and Fitness & 21.3 & $(3.0)$ & 20.4 & $(2.7)$ & 21.0 & $(2.2)$ & 0.61 \\
\hline Salience of Weight/Shape & $20.7 \mathrm{a}$ & $(4.3)$ & $16.5_{\mathrm{b}}$ & (3.3) & $16.9_{\mathrm{b}}$ & (3.3) & $12.60^{-\infty}$ \\
\hline Feelings of Attractiveness & 15.5 & $(1.9)$ & 15.8 & $(1.7)$ & 16.3 & (2.2) & 1.45 \\
\hline Lower Body Fat & $13.8 \mathrm{a}$ & $(2.9)$ & $12.1 \mathrm{a}$ & $(3.0)$ & $9.8_{\mathrm{b}}$ & $(2.5)$ & $19.36^{* * *}$ \\
\hline \multicolumn{8}{|l|}{ Eating behaviours } \\
\hline Restraint & $9.6_{\mathrm{a}}$ & $(5.4)$ & 7.3 & (3.1) & $4.0_{b}$ & $(3.2)$ & 12.74 \\
\hline Disinhibition & 7.7 & $(2.8)$ & 6.2 & $(2,0)$ & 6.3 & $(2.8)$ & $3.75^{*}$ \\
\hline Hunger & 7.7 & $(3.0)$ & $5.7 \mathrm{~s}$ & (2.4) & $8.3_{b}$ & (3.2) & $3.69^{*}$ \\
\hline Perception of current weight & $3.9_{\mathrm{a}}$ & $(0.8)$ & $2.9_{\mathrm{b}}$ & $(0.4)$ & $2.3_{\mathrm{c}}$ & $(0.4)$ & $66.27^{* * * *}$ \\
\hline
\end{tabular}

Note. Means in the same row that do not share subscripts differ at $\mathrm{p}<.05$ according to Tukey's honestly signifficant difference comparison. ${ }^{*} p<.05 .{ }^{*} p<.01 .-p<.001$.

\section{Perception of Current Weight}

There were $19(17 \%)$ participants who perceived themselves to be very overweight, $32(28 \%)$ who thought they were a little overweight, $42(37 \%)$ who thought they were just right, and 20 (18\%) who believed they were a little underweight. No participant suggested that she was very underweight. Means and group differences for perception of current weight are shown in Table 1. Participants desiring a weight loss perceived themselves to be significantly more overweight than both other groups, and participants desiring a weight gain perceived themselves to be significantly thinner than those desiring no change in their weight.

There were relatively strong correlations between perception of weight and both current BMI $(r(113)=.77, p<.001)$ and desired change in BMI $(r(113)=.83, p<.001)$ for the total sample. However, these relationships were much weaker among those participants who expressly wished to lose weight $(r(74)=.56, p<.001, r(74)=.67, p<.001$, respectively). 
Although statistically significant, these correlations indicate that less than one third of variance is shared between perception of weight and actual BMI amongst those who wish to lose weight; approximately $45 \%$ of variance is shared between perception of weight and desired change in BMI. The degree to which perception, actual weight, or degree of desired change was more predictive of body attitudes and eating behaviours was therefore examined further among only those participants who desired a weight loss $(n=74)$.

\section{Table 2}

\section{Correlations Among Key Study Variables for Participants Desiring Weight Loss}

\begin{tabular}{|c|c|c|c|}
\hline & $\begin{array}{l}\text { Current } \\
\text { BMI }\end{array}$ & $\begin{array}{c}\text { Desired change in } \\
\text { BMI }\end{array}$ & $\begin{array}{l}\text { Perception of } \\
\text { current weight }\end{array}$ \\
\hline \multicolumn{4}{|l|}{ Body attitudes } \\
\hline Feelings of Overall Fatness & .22 & $.30^{* *}$ & $.40^{*+*}$ \\
\hline Self-disparagement & .19 & $.31^{* *}$ & $.38^{* * * *}$ \\
\hline Strength and Fitness & -.09 & $-.23^{*}$ & $-.28^{* *}$ \\
\hline Salience of Weight/Shape & -.14 & .05 & $.22^{*}$ \\
\hline Feelings of Attractiveness & -.01 & -.09 & $-.32^{* *}$ \\
\hline Lower Body Fat & $.27^{*}$ & $.34^{* *}$ & $.35^{* * *}$ \\
\hline \multicolumn{4}{|l|}{ Eating behaviours } \\
\hline Restraint & $-.25^{*}$ & -.22 & -.13 \\
\hline Disinhibition & .17 & .21 & .10 \\
\hline Hunger & $.29^{* *}$ & $.25^{*}$ & .15 \\
\hline
\end{tabular}




\section{Relationships Among Attitudes, Behaviours, Perception, Weight, and Desired Weight Loss} Current BMI was largely unrelated to body attitudes, with the exception of a positive association with Consciousness of Lower Body Fat (see Table 2). Body attitudes were more strongly related to the desired change in BMI; significant positive associations were noted for Feelings of Overall Fatness, Self-disparagement, and Consciousness of Lower Body Fat, while there was a significant negative association with Strength and Fitness. However, the strongest and most consistent patterns of association concerning body attitudes were obtained with perception of current weight. Feelings of Overall Fatness, Self-disparagement, Consciousness of Lower Body Fat, and Salience of Weight and Shape were positively associated with the perception of being overweight, while Feelings of Attractiveness and Strength and Fitness demonstrated a negative association with this perception.

Contrary to the above findings, participants' reported eating behaviours were not related to perception of current weight. However, lower levels of Restraint were associated with higher current BMI scores (and vice versa), and higher levels of reported Hunger were positively associated with both current BMI and desired change in BMI (see Table 2). There were few statistically significant relationships between reported eating behaviours and body attitudes. Restrained eating was significantly and positively associated with Feelings of Overall Fatness $(r(74)=.38, p<.001)$ and Salience of Weight and Shape $(r(74)=.46, p<.001)$, while disinhibited eating was significantly more likely to be reported by participants with higher scores for Feelings of Overall Fatness $(r(74)=.43, p<.001)$, and Consciousness of Lower Body Fat $(r(74)=.45, p<.001)$. Hunger was not related to body attitudes.

\section{Attempts To Control Weight}

The conscious attempt to control weight was acknowledged by $58(78 \%)$ of those who sought weight loss. However, $27(36 \%)$ participants who sought weight loss admitted that they commonly overate (rather than eating sensibly), and only $17(23 \%)$ reported having dieted specifically for weight control within the past 12 months. Further, there was no relationship between eating sensibly and the conscious attempt to control weight $\left(\chi^{2}(1, N=74)=0.62\right)$; nor between eating sensibly and having dieted in the past 12 months $\left(\chi^{2}(1, N=74)=0.55\right)$. However, all participants who reported dieting in the past 12 months also acknowledged the conscious attempt to control their weight $\left(\chi^{2}(1, N=74)=4.54, p<.05\right)$.

Participants who admitted usually overeating $(n=27)$ were compared with those who reported usually eating sensibly $(n=47)$. Results are presented in Table 3 . There was a modest difference in perception of current weight; those who usually overate perceived themselves to be more overweight, although there was no significant difference in current BMI. There were also no differences in terms of body attitudes. However, these subgroups did differ on all measures of eating behaviour. Those who usually overate were less likely to report Restraint, but more likely to report Disinhibition and Hunger. 
Table 3

Comparisons of Participants Desiring Weight Loss who Usually Overeat and those who Desire Weight Loss but who Usually Eat Sensibly

\begin{tabular}{|c|c|c|c|c|c|}
\hline & \multicolumn{2}{|c|}{$\begin{array}{l}\text { Usually } \\
\text { overeat }\end{array}$} & \multicolumn{2}{|c|}{$\begin{array}{l}\text { Usually eat } \\
\text { sensibly }\end{array}$} & \multirow[b]{2}{*}{$t$} \\
\hline & $M$ & $(S D)$ & $M$ & $(S D)$ & \\
\hline Current BMI & 22.7 & $(2.2)$ & 22.5 & $(2.6)$ & 0.46 \\
\hline Desired change in BMI & -2.6 & $(1.5)$ & -2.0 & $(1.6)$ & 1.50 \\
\hline Perception of current weight & 4.2 & $(0.8)$ & 3.8 & $(0.7)$ & $2.11^{*}$ \\
\hline \multicolumn{6}{|l|}{ Body attitudes } \\
\hline Feelings of Overall Fatness & 47.8 & $(7.0)$ & 45.5 & $(6.5)$ & 1.39 \\
\hline Self-disparagement & 17.9 & $(4.1)$ & 16.8 & $(3.3)$ & 1.19 \\
\hline Strength and Fitness & 20.4 & $(3.2)$ & 21.8 & $(2.8)$ & 1.91 \\
\hline Salience of Weight/Shape & 21.4 & $(4.6)$ & 20.3 & $(4.1)$ & 1.03 \\
\hline Feelings of Attractiveness & 15.7 & $(2.4)$ & 15.3 & $(1.6)$ & 0.91 \\
\hline Lower Body Fat & 14.4 & $(2.6)$ & 13.4 & $(2.9)$ & 1.40 \\
\hline \multicolumn{6}{|l|}{ Eating behaviours } \\
\hline Restraint & 7.8 & $(4.4)$ & 10.6 & $(5.7)$ & $2.38^{*}$ \\
\hline Disinhibition & 9.1 & $(2.6)$ & 7.0 & $(2.6)$ & $3.42^{* * * *}$ \\
\hline Hunger & 8.9 & $(2.9)$ & 6.9 & $(2.9)$ & $2.88^{* *}$ \\
\hline
\end{tabular}

${ }^{*} p<.05 .{ }^{*} p<.01 . \quad-p<.001$.

Using the data concerning the conscious attempt to control weight and the use of a diet specifically for weight control in the past 12 months, a composite variable was derived for which participants were classified as follows: those who had specifically dieted for weight control in the past year $(n=17)$, those who attempt to control their weight, but had not used a specific diet in the past year $(n=41)$, those who reported not attempting to control their weight $(n=16)$. Differences between these three subgroups, all of whom reported the desire to lose weight, were then explored using the key study variables (see Table 4). 
Table 4

Comparisons of Levels of Behavioural Commitment of Participants Desiring Weight Loss

\begin{tabular}{|c|c|c|c|c|c|c|c|}
\hline & \multicolumn{2}{|c|}{$\begin{array}{c}\text { Has dieted in } \\
\text { past year }\end{array}$} & \multicolumn{2}{|c|}{$\begin{array}{c}\text { Attempts to } \\
\text { control weight }\end{array}$} & \multicolumn{2}{|c|}{$\begin{array}{l}\text { No attempt to } \\
\text { control weight }\end{array}$} & \multirow[b]{2}{*}{$F$} \\
\hline & $M$ & $(S D)$ & $M$ & $(S D)$ & $M$ & $(S D)$ & \\
\hline Current BMI & 22.8 & (1.8) & 22.4 & (2.6) & 22.7 & (2.5) & 0.17 \\
\hline Desired change in BMI & -2.5 & (1.5) & -2.1 & (1.6) & -2.5 & (1.4) & 0.62 \\
\hline Perception of current weight & 4.2 & $(0.7)$ & 3.9 & $(0.8)$ & 3.9 & $(0.6)$ & 1.65 \\
\hline \multicolumn{8}{|l|}{ Body attitudes } \\
\hline Feelings of Overall Fatness & 51.1, & $(5.9)$ & $46.6_{b}$ & $(5.3)$ & $40.6_{c}$ & (6.7) & $13.88^{* *}$ \\
\hline Self-disparagement & $20.2 \mathrm{a}$ & (4.1) & $16.5_{b}$ & (2.9) & $15.8_{b}$ & (3.1) & $9.82^{* * * *}$ \\
\hline Strength and Fitness & 21.0 & $(2.7)$ & 21.6 & (3.3) & 20.8 & (2.5) & 0.53 \\
\hline Salience of Weight/ Shape & $24.2 \mathrm{z}$ & (3.9) & $20.5_{b}$ & (4.1) & $17.6_{\mathrm{c}}$ & (2.5) & $12.81^{* * *}$ \\
\hline Feelings of Attractiveness & $14.1 \mathrm{a}$ & $(2.1)$ & $15.7_{\mathrm{b}}$ & $(1.7)$ & $16.3_{b}$ & (1.6) & $6.89^{* *}$ \\
\hline Lower Body Fat & $15.1_{\mathrm{a}}$ & $(2.3)$ & 13.9 & $(2.8)$ & $12.2_{\mathrm{b}}$ & (2.9) & $4.83^{* * *}$ \\
\hline \multicolumn{8}{|l|}{ Eating behaviours } \\
\hline Restraint & $11.5 \mathrm{a}$ & $(5.6)$ & $10 . \mathrm{a}_{\mathrm{a}}$ & (5.4) & $5.4_{b}$ & (2.9) & $7.49^{* * * *}$ \\
\hline Disinhibition & 9.4a & $(2.8)$ & $7.3_{\mathrm{b}}$ & (2.7) & $7.1_{b}$ & (2.4) & $4.01^{*}$ \\
\hline Hunger & 8.2 & $(3.0)$ & 7.1 & $(2.8)$ & 8.4 & $(3.3)$ & 1.40 \\
\hline
\end{tabular}

Note. Means in the same row that do not share subscripts differ at $p<.05$ according to Tukey's honestly significant difference comparison. $" p<.05 . \quad p<.01 . \quad p<.001$.

Interestingly, these subgroups did not differ significantly in their current BMI, desired change in BMI, or their perception of current weight. However, there were substantial differences in both their body attitudes and their reported eating behaviours. In general, those who had dieted in the past year had a significantly more negative body image than other participants, and reported more dietary restraint and disinhibited eating. Further, those who attempt to control their weight reported higher scores than those who do not in terms of Feelings of Overall Fatness, Salience of Weight and Shape, and Restraint. 


\section{Discussion}

There are two potential effects that Westernisation may have on body image and eating behaviours. On the one hand, Westernisation introduces changes to lifestyle and diet that typically potentiate sedentariness and obesity. Conversely, Westernisation delivers messages that promote unrealistic ideals of thinness and body perfection. That is, Westernisation may engender weight gain (with or without body image disturbance) or body image disturbance (with or without weight gain). It is the ideal of thinness that is prevalent in our sample. While only $9 \%$ of the sample was classified as overweight, $66 \%$ reported the desire to lose at least some weight. This finding is reflective of the result of Lee et al. (1996) who found that $70 \%$ of Chinese undergraduate women surveyed in Hong Kong desired at least modest weight loss. Even if it is acknowledged that the $66 \%$ who desired weight loss in the current sample may have been objectively heavier than the remaining participants, such an overwhelming expressed desire to be thinner potentiates the development of inappropriate attitudinal (negative body image) and behavioural (disordered eating) responses.

Such attitudinal responses were indeed evident when participants desiring weight loss, no change, or weight gain, were compared (Table 1). Participants desiring weight loss felt fatter, were more disparaging of their weight and shape, reported weight and shape to be more salient, and perceived lower body fat to be a particular problem for them. BAQ subscales for which no statistical differences were reported were Strength and Fitness and Feelings of Attractiveness. These results are in line with our previous research identifying that Feelings of Attractiveness is evaluated independently of other body attitudes, while Strength and Fitness is salient to adolescent males but not females (Bond \& McDowell, 2001). The behavioural response of those desiring weight-loss was less clear-cut, although they did report the highest level of Restraint and Disinhibition.

The strength of the attitudinal component of negative body image was further reinforced when participants who reported that weight loss was desirable were subdivided according to the efforts they had employed to effect such a change: dieting in the past year, the conscious attempt to control weight (other than a specific diet), no conscious attempt to control weight (Table 4). Although members of these three groups were equivalent with respect to their current BMI, desired BMI and perception of current weight, they were still identifiably different in terms of their body attitudes. In general, those who had dieted in the past year held the most negative body attitudes.

Of additional concern were the dieters' relatively high levels of Restraint and Dis-inhibition. Restraint is literally the chronic self-control exhibited by many dieters in their attempt to lose weight (Herman \& Polivy, 1980; Polivy \& Herman, 1985), with food intake determined by a balance between the desire to eat and the aspiration to diet (Ruderman, 1985). That is, cognitive processes override normal physiological hunger and satiety cues. While restrained eaters may be successful in adhering to a diet for a time, disinhibiting events (e.g., consumption of forbidden foods, negative emotional states) may interfere with self-control and result in overeating (Lowe, 1993). Commonly this leads to cyclical episodes of restraint and disinhibition with little or no long-term weight loss (Westenhoefer, Broeckmann, Muench, \& Pudel, 1994). Unfortunately, the available data did not allow a more in-depth analysis of this restraint/disinhibition dynamic in the behavioural repertoires of the dieters in our sample, primarily due to the cross-sectional nature of the study. 
Our results provided a far from transparent picture of the relationship between body attitudes and eating behaviours. The single clearest indicator of body attitudes was the participants' perception of their current weight (Table 2). However, neither body attitudes per se, nor perception of current weight, provided notable predictive power in relation to eating behaviours. This finding is reinforced by the data presented in Table 3; self-reported eating pattern was consistently associated with Restraint, Disinhibition and Hunger, but was not guided by body attitudes at all. In summary, these data are suggestive of a mismatch between participants' attitudes and behaviours. It can be inferred that while attitudes generally tended towards slimness, behaviours were not greatly influenced, except for those with the greatest desire to lose weight (i.e., those with the most entrenched attitudes).

Two interpretations of these findings are offered. First, Westernisation has influenced attitudes but not flowed through to behaviours. The extent of the influence of Westernisation is difficult to objectively determine, but it is clear that other authors do suggest this is on the increase in the region (Rahim, 1998; Robinson \& Goodman, 1996). Moreover, it is of particular relevance to the current sample as they are likely to represent the most Westernised of Malay adolescents. They are highly educated, fluent in English, comprise the demographic frequently targeted by Western marketeers, and have been prepared for study overseas. However the potential moderating influence of entrenched religious and cultural patterns cannot be underestimated. By definition, the members of our sample were Muslim. Muslims uphold regid dietary restrictions, such as the requirement to eat Halal food. It is therefore possible that, while having disordered body attitudes, participants were to some extent protected from the behavioural manifestation of these attitudes by these strict practices. This supports the assertion of Indran and Hatta (1995) that sociocultural traditions govern food intake, thus negating the effects of Westernisation.

A second explanation is that the observed results may simply reflect the attitude-behaviour inconsistency that prevails in many investigations of social cognition. It is now widely accepted that the general relationship between attitudes and behaviours is at best modest unless certain conditions (e.g., compatibility and specificity) are met (Ajzen \& Fishbein, 1980; Eagly \& Chaiken, 1993). Therefore it is not surprising that the negative body image construct at the attitudinal level was only weakly associated with eating behaviours that might characterise a disturbed eating pattern. Additionally, it is accepted from the work of social learning theorists that behaviour can be learned but not necessarily expressed (Bandura, 1986). That is, it is possible for a negative body image to be reported without, necessarily, the exhibition of the full repertoire of behaviours normally associated with disturbed eating patterns (Restraint, Disinhibition, etc.). In this sense the behavioural potential exists for a clinical disorder to develop. Situations that might precipitate such a manifestation include periods of psychological and physical stress, and increased exposure to concepts that reinforce the predisposition. These factors are specifically relevant to the current sample who have left Malaysia to study in Australia, thus being subject to increased stress as they adjust to a more Western lifestyle. This phenomenon, in which host Western cultural ideals dominate introduced cultures, has been termed "sojourner adjustment" (Ward \& Kennedy, 1993), that is indicative of a general trend towards a global culture of modernity (Katzman \& Lee 1997; Lee, 1996; Littlewood, 1995). 
In conclusion, our findings imply that at least one group of young Malay women may be susceptible to body image disturbance. However, our data must be considered exploratory at best. The current participants were from the educated classes and had recently relocated to Australia, therefore experiencing a higher level of exposure to Westernisation than their peers in Malaysia. We must not underestimate the degree to which attitudes to food and an understanding of the diet-health link are socially and culturally specific (Rozin, Fischler, Imada, Sarrubin \& Wrzesniewski, 1999). It remains to be determined, for example, whether our findings are relevant to Malays resident in Malaysia, Malays with broader educational and socio-economic backgrounds than represented in our sample, and indeed members of other Malaysian ethnic groups. One issue of interest is a Malaysian perspective on the relevance of body mass index, particularly the validity of classifications such as 'normal' and 'overweight' in the Asian context. Indeed it is likely that similar methodological considerations and cultural conceptions of body image need to be acknowledged in investigations with women in other Asian and Pacific countries. It is suggested that such research be conducted by local researchers, who have the appropriate cultural understanding of the issues at hand, and a greater insight as to the best way to design broad-scale interventions should they be required.

\section{References}

Ajzen, I., \& Fishbein, M. (1980). Understanding attitudes and predicting social behavior. Englewood Cliffs, NJ: Prentice-Hall.

Altabe, M. N. (1996). Issues in the assessment and treatment of body image disturbance in culturally diverse populations. In J. K. Thompson (Ed.), Body image, eating disorders, and obesity (pp. 129-147). Washington, DC: American Psychological Association.

Ariffin, J. (1995). At the crossroads of rapid development: Malaysian society and anomie. International Journal of Sociology and Social Policy, 15(8-10), 343-371.

Australian Psychological Society. (1994). Code of professional conduct. Melbourne: The Society.

Bandura, A. (1986). Social foundations of thought and action: A cognitive social theory. Englewood Cliffs, NJ: Prentice-Hall.

Banks, C. G. (1992). 'Culture' in culture-bound syndromes: The case of anorexia nervosa. Social Science and Medicine, 34, 867-884.

Ben-Tovim, D. I., \&Walker, M. K. (1991). The development of the Ben-Tovim Walker Body Attitudes Questionnaire (BAQ), a new measure of women's attitudes towards their own bodies. Psychological Medicine, 21, 775-784.

Bond, M. J., \& McDowell, A. J. (2001). An adolescent conception of body image and weight loss behaviours. Journal of Applied Health Behaviour, 3(2), 8-15.

Buhrich, N. (1981). Frequency and presentation of anorexia nervosa in Malaysia. Australian and New Zealand Journal of Psychiatry, 15, 153-155.

Button, E. J., \& Whitehouse, A. (1981). Subclinical anorexia nervosa. Psychological Medicine, 11, 509-516.Crawford, D. A., \& Worsley, A. (1988). Dieting and slimming practices of South Australian women. Medical Journal of Australia, 148, 325-331.

Cronbach, L. J. (1951). Coefficient alpha and the internal structure of tests. Psychometrika, 16, 297334.

Dolan, B. (1991). Cross-cultural aspects of anorexia nervosa and bulimia: A review. International Journal of Eating Disorders, 10, 67-79. Jovanovich.

Eagly, A. H., \& Chaiken, S. (1993). The psychology of attitudes. Fort Worth, TX: Harcourt Brace

Felts, W. M., Parrillo, A. V., Chenier, T., \& Dunn, P. (1996). Adolescents' perceptions of relative weight and self-reported weight-loss activities: Analysis of 1990 YRBS national data. Jourmal of Adolescent Health, 18, 20-26.

Furnham, A., \& Baguma, P. (1994). Cross-cultural differences in the evaluation of male and female body shapes. International Journal of Eating Disorders, 15, 81-89.

Garrow, J.S., \& Webster, J. (1985). Quetelet's Index $\left(\mathrm{W} / \mathrm{H}^{2}\right)$ as a measure of fatness. International Journal of Obesity, 9, 147-153. 
Goh, S. E., Ong, S. B., \& Subramaniam, M. (1993). Eating disorders in Hong Kong [Letter]. British Journal of Psychiatry, 162, 276-277.

Heinberg, L. J. (1996). Theories of body image disturbance: Perceptual, developmental, and sociocultural factors. In J. K. Thompson (Ed.), Body image, eating disorders, and obesity (pp. 27-47). Washington, DC: American Psychological Association.

Herman, C. P., \& Polivy, J. (1980). Restrained eating. In A. J. Stunkard (Ed.), Obesity (pp. 208-225). Philadelphia: W.B. Saunders.

Hughes, K., Yeo, P. P. B., Lun, K. C., Thai, A. C., Wang, K. W., \& Cheah, J. S. (1990). Obesity and body mass indices in Chinese, Malays and Indians in Singapore. Annals of the Academy of Medicine, Singapore, 19, 333-338.

Indran, S. K., \& Hatta, M. (1995). Eating attitudes among adolescent girls in a Malaysian secondary school using the EAT questionnaire. International Journal of Social Psychiatry, 41, 299-302.

Kahn, J. S. (1997). Malaysian modern or anti-anti Asian values. Thesis Eleven, 50(Aug.), 15-33.

Katzman, M. A., \& Lee, S. (1997). Beyond body image: The integration of feminist and transcultural theories in the understanding of self-starvation. International Journal of Eating Disorders, 22, 385-394.

Kenny, D., \& Adams, R. (1994). The relationship between eating attitudes, body mass index, age, and gender in Australian university students. Australian Psychologist, 29, 128-134.

Kiriike, N., Nagata, T., Sirata, K., \& Yamamoto, N. (1998). Are young women in Japan at high risk of eating disorders: Decreased BMI in young females from 1960 to 1995. Psychiatry and Clinical Neurosciences, $52,279-281$.

Kling, Z. (1995). The Malay family: Beliefs and realities. Journal of Comparative Family Studies, 26,

43-66.

Laderman, C. (1984). Food ideology and eating behaviour: Contributions from Malay studies. Social Science \& Medicine, 19, 547-559.

Lee, S. (1994). The Diagnostic Interview Schedule and anorexia nervosa in Hong Kong. Archives of General Psychiatry, 51, 251-252.

Lee, S. (1996). Reconsidering the status of anorexia nervosa as a Western culture-bound syndrome. Social Science and Medicine, 42, 21-34.

Lee, S. (1998). Global modernity and eating disorders in Asia. European Eating Disorders Review, 6 , 151-153.

Lee, S., Leung, T., Lee, A. M., Yu, H., \& Leung, C. M. (1996). Body dissatisfaction among Chinese undergraduates and its implications for eating disorders in Hong Kong. International Journal of Eating Disorders, 20, 77-84,

Lim, M. K., Liam, B. L., Ng, D., \& Teow, R. (1994). Twenty-five years of national service - changes in height, weight and body mass index. Annals of the Academy of Medicine, Singapore, 23, 770-774.

Lippincott, J. A., \& Hwang, H. S. (1999). On cultural similarities in attitudes toward eating of women students in Pennsylvania and South-Korea. Psychological Reports, 85, 701-702.

Littlewood, R. (1995). Psychopathology and personal agency: Modernity, culture change and eating disorders in South Asian societies. British Journal of Medical Psychology, 68, 45-63.

Lowe, M. R. (1993). The effects of dieting on eating behavior: A three-factor model. Psychological Bulletin, 114, 100-121.

Maude, D., Wertheim, E. H., Paxton, S., Gibbons, K., \& Szmukler, G. (1993). Body dissatisfaction, weight loss behaviours, and bulimic tendencies in Australian adolescents with an estimate of female data representativeness. Australian Psychologist, 28, 128-132.

Nain, Z. (1996). The impact of the international marketplace on the organisation of Malaysian television. In D. French \& M. Richards (Eds.), Contemporary television: Eastern perspectives (pp. 157-180). New Delhi: Sage.

Nogami, Y. (1997). Eating disorders in Japan: A review of the literature. Psychiatry and Clinical Neurosciences, 51, 339-346.

Norušis, M. J. (1993). SPSS for Windows: Base system user's guide, release 6.0. SPSS Inc., Chicago.

Pate, J. E., Pumariega, A. J., Hester, C., \& Garner, D. M. (1992). Cross-cultural patterns in eating disorders: A review. Journal of the American Academy of Child and Adolescent Psychiatry, 31, 802-809.

Patton, G. C., Johnson-Sabine, E., Wood, K., Mann, A. H., \& Wakeling, A. (1990). Abnormal eating attitudes in London schoolgirls - a prospective epidemiological study: Outcome at twelve month follow-up. Psychological Medicine, 20, 383-394. 193-201.

Polivy, J., \& Herman, C.P. (1985), Dieting and binging: A causal analysis. American Psychologist, 40, 
Powell, A. D., \& Kahn, A. S. (1995). Racial differences in women's desires to be thin. International Journal of Eating Disorders, 17, 191-195.

Rahim, L. Z. (1998). In search of the 'Asian way': Cultural nationalism in Singapore and Malaysia. Commonwealth and Comparative Politics, 36(3), 54-73.

Rand, C. S. W., \& Kuldau, J. M. (1990). The epidemiology of obesity and self-defined weight problem in the general population: Gender, race, age, and social class. International Joumal of Eating Disonders, 9, 329343.

Robinson, R., \& Goodman, D. S. G. (Eds.). (1996). The new rich in Asia: Mobile phones, McDonalds, and middle-class revolution. London: Routledge.

Rodin, J., Silberstein, L., \& Striegel-Moore, R. (1985). Women and weight: A normative discontent. In T. B. Sonderegger (Ed.), Nebraska Symposium on Motivation: Vol. 32. Psychology and Gender (pp. 267-307). Lincoln, NB: University of Nebraska Press.

Rosen, J. C., \& Gross, J. (1987). Prevalence of weight reducing and weight gaining in adolescent girls and boys. Health Psychology, 6, 131-147.

Rozin, P., Fischler, C., Imada, S., Sarubin, A., \& Wrzesniewski, A. (1999). Attitudes to food and the role of food in life in the U.S.A., Japan, Flemish Belgium and France: Possible implications for the diet-health debate. Appetite, 33, 163-180.

Ruderman, A.J. (1985). Dysphoric mood and overeating: A test of restraint theory's disinhibition hypothesis. Journal of Abnormal Psychology, 94, 78-85.

Rumpel, C., \& Harris, T. B. (1994). The influence of weight on adolescent self-esteem. Journal of Psychosomatic Research, 38, 547-556.

Schmidt, U. (1993). Bulimia nervosa in the Chinese. International Joumal of Eating Disoders, 14, 505-

509.

Stevens, J., Kumanyika, S. K., \& Keil, J. E. (1994). Attitudes toward body size and dieting: Differences between elderly Black and White women. American Journal of Public Health, 84, 1322-1325.

Stunkard, A. J. (1997). Eating disorders: The last 25 years. Appetite, 29, 181-190.

Stunkard, A. J., \& Messick, S. (1985). The three-factor eating questionnaire to measure dietary restraint, disinhibition and hunger. Journal of Psychosomatic Research, 29, 71-83.

Vandereycken, W. (1993). The sociocultural roots of the fight against fatness: Implications for eating disorders and obesity. Eating Disorders, 1, 7-16.

Ward, C. \& Kennedy, A. (1993). Where's the 'culture' in cross-cultural transition?: Comparative studies of sojourner adjustment. Journal of Cross-Cultural Psychology, 24, 221-249.

Westenhoefer, J., Broeckmann, P., Muench, A. K., \& Pudel, V. (1994). Cognitive control of eating behaviour and the disinhibition effect. Appetite, 23, 27-41.

Wilkinson, J. Y., Ben-Tovim, D. I., \& Walker, M. K. (1994). An insight into the personal and cultural significance of weight and shape in large Samoan women. International Journal of Obesity, 18, 602-606.

\begin{abstract}
Author Notes
Andrew J. McDowell and Malcolm J. Bond, Department of Psychiatry.

Correspondence concerning this article should be addressed to Dr Malcolm Bond, Department of Psychiatry, School of Medicine, Flinders University, GPO Box 2100, Adelaide, Australia, 5001. Electronic mail may be sent via Internet to malcoimbond@flinders.eduau.
\end{abstract}

Manuscript originally submitted November 2001.

Revised and accepted November 2002. 